\title{
Mapas cognitivos na avaliação da Aprendizagem Baseada em Problemas
}

\author{
Adriana Casale, Nídia P. Kuri \& Antônio N. R. da Silva \\ Universidade de São Paulo, Brasil
}

Resumo

Este estudo tem por objetivo investigar o aproveitamento dos estudantes do Curso de Engenharia Civil em que foi aplicado o método Aprendizagem Baseada em Problemas - Problem Based Learning (PBL) como parte de inovações pedagógicas implementadas em uma disciplina de Engenharia de Transportes. Para analisar o aproveitamento da amostra submetida às alternativas instrucionais foram utilizadas as respostas dos estudantes a um questionário para avaliação do aprendizado, elaborado pelo professor. Este instrumento contribuiu para avaliar e comparar o aprendizado da amostra com uma turma de alunos submetida ao modelo tradicional de ensino. As respostas foram agrupadas e analisadas por meio de mapas cognitivos, contribuindo para a investigação dos efeitos do método PBL e da ocorrência de aproveitamentos diferenciados entre os alunos. Os resultados indicam que os estudantes obtiveram maior envolvimento, desenvolvimento de comportamentos e atitudes, comprometimento com a própria aprendizagem e um aproveitamento significativamente melhor com o novo desenho da disciplina.

Palavras-chave

Aprendizagem Baseada em Problemas; Mapas cognitivos; Ensino; Avaliação

\section{Introdução}

A adaptação contínua, por parte dos indivíduos, para acompanhar as transformações, em especial as tecnológicas, tem se tornado cada vez mais 
necessária. No campo da educação, esse fato é ainda mais evidente, sobretudo nas instituições de ensino superior que formam profissionais que atuarão em um mercado de trabalho bastante competitivo e exigente quanto aos conhecimentos, competências e habilidades requeridas de seus profissionais.

A educação em Engenharia também está sob intensa pressão por mudanças. Um fator predominante, que requer dos engenheiros uma atualização constante de suas técnicas e conhecimentos, está relacionado aos conhecimentos científicos e tecnológicos que fundamentam sua formação acadêmica. Incessantemente gerados e modificados, muitas vezes esses conhecimentos se tornam obsoletos ainda durante o período de formação universitária, exigindo frequentes atualizações para que possam ser utilizados na vida profissional. Adequar o ensino de Engenharia e a formação do futuro engenheiro às inovações e imposições do mercado de trabalho tem sido um desafio para as escolas de engenharia de todo o mundo. Docentes, pesquisadores e estudiosos de muitas instituições de ensino superior, como as de educação em Engenharia (Dochy et al., 2003; Yusof et al., 2004; Hassan, et al., 2004; Yusof et al., 2005; Keating \& Gabb, 2006), buscam adequar ou reestruturar seus cursos com o objetivo de proporcionar formação adequada aos futuros profissionais.

No Brasil, alguns docentes e pesquisadores (Kuri, et al., 2006; Kuri, et al., 2007; Kalatzis, 2008) também têm realizado investigações na busca por alternativas pedagógicas, visando o aperfeiçoamento do processo de ensino e aprendizagem, a formação de engenheiros críticos, conscientes de sua atuação como cidadãos, capazes de inovar, produzir conhecimento e enfrentar questões sociais e profissionais.

Uma das alternativas pedagógicas, implementadas em uma disciplina da Engenharia Civil, refere-se ao uso do método Aprendizagem Baseada em Problemas - Problem Based Learning (PBL), em uma plataforma de ensino a distância, apoiada nas preferências de aprendizagem e de personalidade dos estudantes (Kuri, et al., 2007; Rodrigues da Silva, 2008). Essa nova proposta pedagógica, agregada ao conteúdo programático da disciplina, constitui-se no ponto de partida para a realização deste estudo. Entendendo que a adoção de alternativas instrucionais pode aperfeiçoar o processo de ensino e aprendizagem, a proposta do presente estudo é investigar o aproveitamento 
dos estudantes envolvidos com a experiência didática em uma disciplina do Curso de Engenharia Civil, especialmente no que se refere ao uso do PBL como método de aprendizagem na Engenharia.

Avaliações dessa natureza são frequentemente baseadas em critérios quantitativos de desempenho dos alunos, como demonstrado no estudo de Kalatzis (2008) e no trabalho de Kuri, et al. (2006). Este tipo de avaliação permite análises rigorosas do ponto de vista estatístico, mas não é capaz de captar aspectos qualitativos da formação dos estudantes. Por este motivo, adotou-se na presente pesquisa a técnica Análise e Desenvolvimento de Opções Estratégicas - Strategic Options Development and Analysis (SODA) - para a construção de mapas cognitivos. O mapeamento cognitivo, como forma de avaliar a aprendizagem, possibilitou investigar de maneira mais aprofundada o aproveitamento dos estudantes, bem como os efeitos do método PBL na aquisição de conhecimentos e no desenvolvimento de atitudes.

\section{Fundamentação teórica}

O PBL foi constituído como método no início dos anos de 1970, com os estudos de Barrows e Tamblyn na escola de medicina da Universidade de McMaster no Canadá (Barrows \& Tamblyn, 1976; Rehm, 1998; Subramaniam, 2006). Os pesquisadores desenvolveram o PBL na tentativa de suprir as dificuldades identificadas no ensino de graduação do curso de medicina (Barrows \& Tamblyn, 1980; White, 1996). Conforme Ribeiro (2005), os estudantes concluíam o curso com muitos conceitos, mas com poucos comportamentos e estratégias associadas à aplicação de informações a um diagnóstico.

Os atributos de um método de aprendizagem "ideal" incluem aquisição do conhecimento profissional, desenvolvimento do pensamento crítico, habilidades para a resolução de problemas e uma aprendizagem duradoura, gerando uma melhor formação profissional e pessoal. Neste sentido, o PBL enfatiza a aprendizagem em vez da instrução, além de ser um método centrado no aluno. Ele permite que o estudante aprenda a partir de um problema proposto, real ou simulado, interagindo, obtendo dados, formulando hipóteses, tomando decisões e emitindo julgamento. Assim, o aluno torna-se responsável por sua própria aprendizagem. 
Por esta razão, Savery e Duffy (1995) apontam que o PBL, como um modelo geral inicialmente desenvolvido para o ensino de Medicina, tem sido refinado e implementado em diversas Escolas. Nas áreas relacionadas à saúde, suas aplicações mais difundidas ocorrem, frequentemente, nos primeiros anos do currículo, em substituição às aulas expositivas tradicionais. De acordo com esses autores, esse modelo não se restringiu apenas aos programas curriculares das universidades de Medicina. Ele foi adotado em outras áreas, incluindo a administração, enfermagem (Milter \& Stinson, 1993), pedagogia (Bridges \& Hallinger, 1992; Duffy, 1994), engenharia, arquitetura, direito, serviço social (Boud \& Feletti, 1991) e, ainda, em outros níveis da educação, como no ensino fundamental e médio (Barrows, 2008; Barrows \& Myers, 1993). Ademais, no século XX, estendeu-se por países como Austrália, Holanda, Nova Zelândia e, hoje, muitas universidades e escolas têm seus programas desenvolvidos a partir do PBL.

No Brasil, além do curso de Medicina iniciado no ano de 2007 na Universidade Federal de São Carlos, que utiliza o PBL como método de ensino e aprendizagem, há algumas poucas universidades e cursos que adotaram esse método, tal como relatado por Ribeiro (2005). Entretanto, embora as iniciativas ainda sejam escassas, mostrando a necessidade de pesquisas envolvendo o PBL no contexto brasileiro, é possível verificar resultados significativos onde foi implementado. Ademais, é um método adaptável para uma variedade de ambientes educacionais (Barrows, 2008), razão pela qual existe uma profusão de definições e interpretações a ele relativas. Este estudo apoia-se nos preceitos de Howard Barrows, um dos pioneiros no desenvolvimento do método PBL.

Conforme Pawson et al. (2006), desde que os problemas não respeitem os limites disciplinares, o PBL frequentemente envolve colaboração entre as disciplinas. Isso requer dos estudantes a integração dos conhecimentos, de diversos assuntos e de várias disciplinas. Essa experiência intensifica as formas de conduzir, sintetizar ou de aprender como se aprende, em vez de assimilar o conteúdo antes da sua aplicação completa.

Muitas pesquisas têm mostrado a eficiência do método PBL na melhoria do aproveitamento na aprendizagem. Dochy et al. (2003) realizaram uma meta-análise dos efeitos do PBL no desempenho de estudantes do ensino superior. Os resultados fornecidos por 43 estudos empíricos, revisados 
por esses autores, sugerem que o método produz efeitos bastante positivos, tanto em relação ao desenvolvimento de habilidades nos estudantes, quanto ao desempenho na aplicação dos conhecimentos adquiridos. Para Yusof et al. (2005), o uso do PBL é recomendado na Engenharia, particularmente, porque promove a aprendizagem profunda e desenvolve habilidades de resolução de problemas, além de trazer melhorias significativas no que se refere ao desenvolvimento de habilidades gerais e promoção de atitudes positivas entre os estudantes.

No entanto, experiências realizadas com o método PBL apontam para a necessidade da mudança de hábito, tanto de estudantes quanto de professores, em virtude dos requisitos relacionados ao método para lidar com estratégias de aprendizagem ativa e de demandar uma maior disposição para a aprendizagem autônoma. Segundo observações de Hassan et al. (2004), alguns estudantes sentem dificuldade em tornarem-se pensadores críticos ativos e alguns professores podem enfrentar dificuldades no desempenho do seu papel de tutor, facilitador das discussões, administrador dos grupos de trabalho ou, ainda, de desafiador do pensamento crítico dos estudantes. Os autores também mencionam algumas dificuldades relacionadas ao uso do método PBL, principalmente no que diz respeito à limitada experiência de administração dos grupos, falta de familiaridade com a pesquisa e de retorno do conhecimento na aprendizagem.

O estudo de Kalatzis (2008) aponta vantagens na adoção do método $\mathrm{PBL}$ no que se refere às notas dos estudantes. Utilizando uma abordagem quantitativa, por meio de um teste de médias, comprova o desenvolvimento de maiores níveis de aproveitamento de estudantes de engenharia que vivenciaram o PBL em uma disciplina do curso de Engenharia Civil. O resultado pode ser observado na Tabela 1. 


\begin{tabular}{|c|c|c|c|c|c|c|}
\hline \multirow{2}{*}{$\begin{array}{l}\text { Métodos } \\
\text { PBL }\end{array}$} & \multirow{2}{*}{$\begin{array}{c}\text { Alunos } \\
29\end{array}$} & \multirow{2}{*}{$\begin{array}{l}\text { Média } \\
6.568\end{array}$} & \multirow{2}{*}{$\begin{array}{c}\begin{array}{c}\text { Erro } \\
\text { Padrão }\end{array} \\
0.1866\end{array}$} & \multirow{2}{*}{$\begin{array}{c}\begin{array}{c}\text { Desvio } \\
\text { Padrão }\end{array} \\
1.005\end{array}$} & \multicolumn{2}{|c|}{$\begin{array}{c}\text { [Intervalo de } \\
\text { Confiança - 95\%] }\end{array}$} \\
\hline & & & & & 6.186 & 6.951 \\
\hline Tradicional & 26 & 5.2 & 0.3054 & 1.557 & 4.570 & 5.829 \\
\hline Conjunto & 55 & 5.921 & 0.1964 & 1.457 & 5.5279 & 6.315 \\
\hline Diferença & - & 1.3689 & 0.3498 & - & 0.6672 & 2.0707 \\
\hline \multicolumn{4}{|c|}{$\operatorname{diff}=\operatorname{mean}(0)-\operatorname{mean}(1)$} & & \multicolumn{2}{|c|}{$t=-3.9127$} \\
\hline \multicolumn{4}{|l|}{ Ho: diff $=0$} & \multicolumn{3}{|c|}{ degrees of freedom $=53$} \\
\hline \multicolumn{2}{|l|}{ Ha: diff $<0$} & \multicolumn{2}{|l|}{ Ha: diff $!=0$} & & \multicolumn{2}{|c|}{ Ha: diff $>0$} \\
\hline \multicolumn{2}{|c|}{$\operatorname{Pr}(T<t)=0.0001$} & \multicolumn{2}{|c|}{$\operatorname{Pr}(|\mathrm{T}|>|\mathrm{t}|)=0.0003$} & & \multicolumn{2}{|c|}{$\operatorname{Pr}(T>t)=0.9999$} \\
\hline
\end{tabular}

Tabela 1 - Teste de médias entre as turmas

Conforme apresentado na Tabela 1, o resultado, considerado estatisticamente significativo, indica que os estudantes que aprenderam pelo PBL obtiveram um acréscimo de 1.368 na média, em comparação com os alunos que aprenderam pelo método convencional. Essa menor variabilidade aponta que o uso do método PBL reduz a heterogeneidade das notas dos alunos.

No presente estudo, a metodologia empregada e os resultados do uso do PBL na avaliação da aprendizagem estão relatados nos próximos itens.

\section{Metodologia}

Os dados utilizados neste trabalho foram obtidos com uma amostra composta por 30 (trinta) estudantes do curso de Engenharia Civil da Escola de Engenharia de São Carlos da Universidade de São Paulo, representantes de duas turmas que frequentaram uma disciplina que trata de Planejamento de Transportes, no segundo semestre do ano 2006, usualmente ministrada por dois docentes, cada um responsável por uma turma. Deste total, 12 (doze) estudantes vivenciaram o PBL como parte da nova proposta pedagógica implementada por um dos docentes na disciplina - experiência descrita com detalhes em Kuri, et al. (2007) e Kalatzis (2008) - e 18 (dezoito) cumpriram a disciplina ministrada pelos métodos "tradicionais" de ensino.

Para investigar se a adoção do método PBL resultou em um melhor aproveitamento dos alunos na disciplina, um questionário, idealizado pelo docente responsável pela nova proposta pedagógica, foi aplicado aos 
estudantes da amostra no primeiro semestre de 2008 - portanto, mais de um ano após a conclusão da disciplina. O instrumento compõe-se de duas partes. A Parte 1 está subdividida em três tópicos (Questões 1a, 1b e 1c) decorrentes da teoria sobre planejamento e análise de sistemas de transportes. A Parte 2 propõe dois problemas (Questões $2 a$ e $2 b$ ) que envolvem a aplicação prática dos conhecimentos e técnicas abordadas na disciplina concluída em 2006. As Questões 1a, 1b e 1c foram respondidas pelos estudantes em sala de aula e as Questões 2a e 2b no laboratório de informática, com acesso à Internet para pesquisa. As questões propostas aos estudantes são apresentadas no Quadro 1.

Parte 1) O que você entende por:

a. Planejamento de sistemas de transportes;

b. Análise de sistemas de transportes;

c. Sistemas de transportes.

Parte 2) Parabéns! Você acaba de chegar, com outros cinquenta candidatos, à última fase do processo seletivo para concorrer a UMA vaga de Engenheiro de uma grande empresa que atua no ramo de Logística, localizada em Campinas, no estado de São Paulo. Essa etapa é decisiva para a escolha do profissional a ser contratado e será baseada na avaliação da(s) alternativa(s) que você apresentar para os dois seguintes problemas:

a) Há uma demanda localizada na cidade de Milagres, na Bahia, por um medicamento específico que é produzido apenas em Campinas e tem validade de 24 horas. Sabendo que devem ser fornecidas doses regulares desse medicamento nos próximos 15 dias, com entrega de uma caixa de 5 quilos por dia, como você faria o transporte desse produto e quanto você imagina que esse transporte poderia custar para o comprador?

b) A empresa pretende candidatar-se a um edital que prevê a entrega de todo o suprimento de álcool combustível necessário para atender à cidade de Manaus nos próximos 5 anos. Que alternativas e custos você imagina que a empresa deve apresentar para concorrer a esse contrato, sabendo que o álcool encontra-se disponível na cidade de Paulínia, estado de São Paulo?

\section{Quadro 1 - Questionário para avaliação do aprendizado}

A primeira questão foi estruturada de modo a que os estudantes demonstrassem seu entendimento com relação aos três tópicos solicitados e 
as respostas possibilitassem a captura dos conceitos centrais tratados na disciplina. Os problemas ( $a$ e b), propostos na segunda parte, foram formulados para permitir que os alunos explorassem as técnicas e conhecimentos intrínsecos à disciplina. A partir das respostas, fornecidas pelos estudantes, procedeu-se à construção de mapas cognitivos mediante 0 uso da técnica Análise e Desenvolvimento de Opções Estratégicas - Strategic Options Development and Analysis (SODA), que permite o agrupamento de diversos mapas, possibilitando uma melhor organização e visualização das informações neles contidas. A técnica empregada e a construção desses mapas estão fundamentadas nos conceitos de Eden e Simpson (1989) sobre mapas cognitivos.

Dentre as diversas possibilidades de uso, o mapeamento cognitivo pode ser visto como uma tentativa de isolar e representar os constructos de um indivíduo e dispô-los de maneira hierarquizada. Neste sentido, um mapa pode ser visto como uma rede de ideias que, capturadas diretamente daquele que as expressa, são conectadas para refletir a forma como, na perspectiva desse indivíduo, elas se relacionam. Os mapas apresentados neste estudo representam de forma sintética a estrutura e conteúdo dos assuntos tratados na disciplina, na percepção dos alunos. Foram construídos com o intuito de investigar os efeitos do método PBL no aproveitamento dos estudantes, mediante a comparação das respostas fornecidas pelos participantes e não participantes da experiência. Os conceitos dispostos nos mapas expressam os entendimentos, explicações, estratégias e dados utilizados pelos estudantes, ao passo que as ligações entre conceitos são representadas por setas, que indicam como um conceito conduz ou tem implicação sobre outro. Sabendo que os conceitos refletem, de modo geral, o entendimento dos alunos sobre as questões abordadas, os mapas estão traçados de maneira a fluírem do centro para as extremidades.

No mapeamento das respostas às questões abordadas na Parte 1 do instrumento de avaliação (Questões 1a, 1b, 1c), foi adotado um sistema para facilitar a identificação de seus tópicos. Assim, nos mapas referentes à Parte 1, a questão sobre planejamento de sistemas de transportes (Questão 1a) é identificada em itálico (Planejamento), a que se refere à análise de sistemas de transportes (Questão 1b) é destacada com o uso do negrito (Análise), e a referente aos sistemas de transportes (Questão 1c) é escrita em letras 
maiúsculas (SISTEMAS DE TRANSPORTES). Ademais, há casos em que os conceitos envolvem os três tópicos, sendo representados pelos três recursos mencionados, simultaneamente. No mapeamento das respostas relacionadas à Parte 2, as referentes à Questão 2a estão localizadas na parte superior das figuras, ao passo que as relativas à Questão $2 \mathrm{~b}$ se situam na parte inferior. Os resultados do mapeamento cognitivo são analisados e discutidos na sequência.

\section{Resultados: mapas cognitivos e aproveitamento dos estudantes}

A Figura 1 representa o entendimento dos estudantes sobre os tópicos $1 \mathrm{a}, 1 \mathrm{~b}$ e 1c da Parte 1 e refere-se às respostas fornecidas pelo grupo exposto às inovações pedagógicas. Nota-se, por meio das respostas expressas no mapa, que, em linhas gerais, os estudantes apreenderam os conceitos mais gerais e abrangentes da disciplina, apontando seus significados, funções, finalidades e exemplos de utilização, principalmente no que se refere ao planejamento, análise e sistemas de transportes. Pode-se observar ainda que o nível de abrangência e profundidade na forma como as questões foram respondidas, apontado por Barrows (1996) como fundamental na resolução de problemas, sugere que o método PBL exerceu um efeito positivo nas maneiras encontradas pelos estudantes para solucionarem os problemas. 


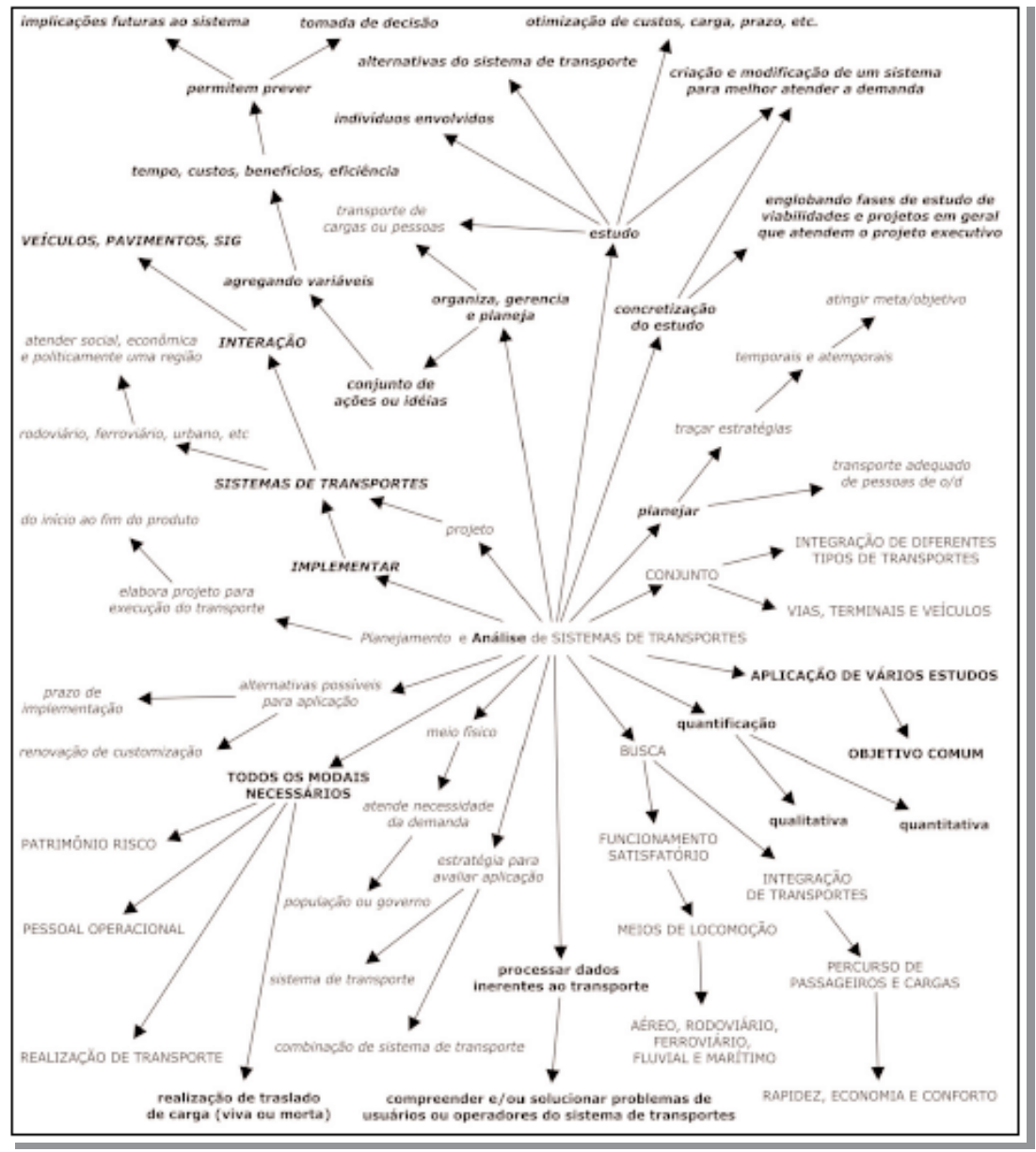

Figura 1 - Mapa cognitivo dos estudantes submetidos às inovações pedagógicas

Legenda: Planejamento (itálico)

Análise (negrito)

Sistemas (MAIÚSCULAS)

As respostas dos estudantes que frequentaram a disciplina ministrada do modo "tradicional" estão representadas na Figura 2. Observa-se que não 
parece ter havido diferenças entre as respostas dos estudantes em relação à teoria. Independentemente do formato em que as aulas ocorreram, o método convencional de ensino possibilita o desenvolvimento de técnicas de memorização, fazendo com que os estudantes respondam às questões mesmo sem a apreensão dos conceitos nelas envolvidos.

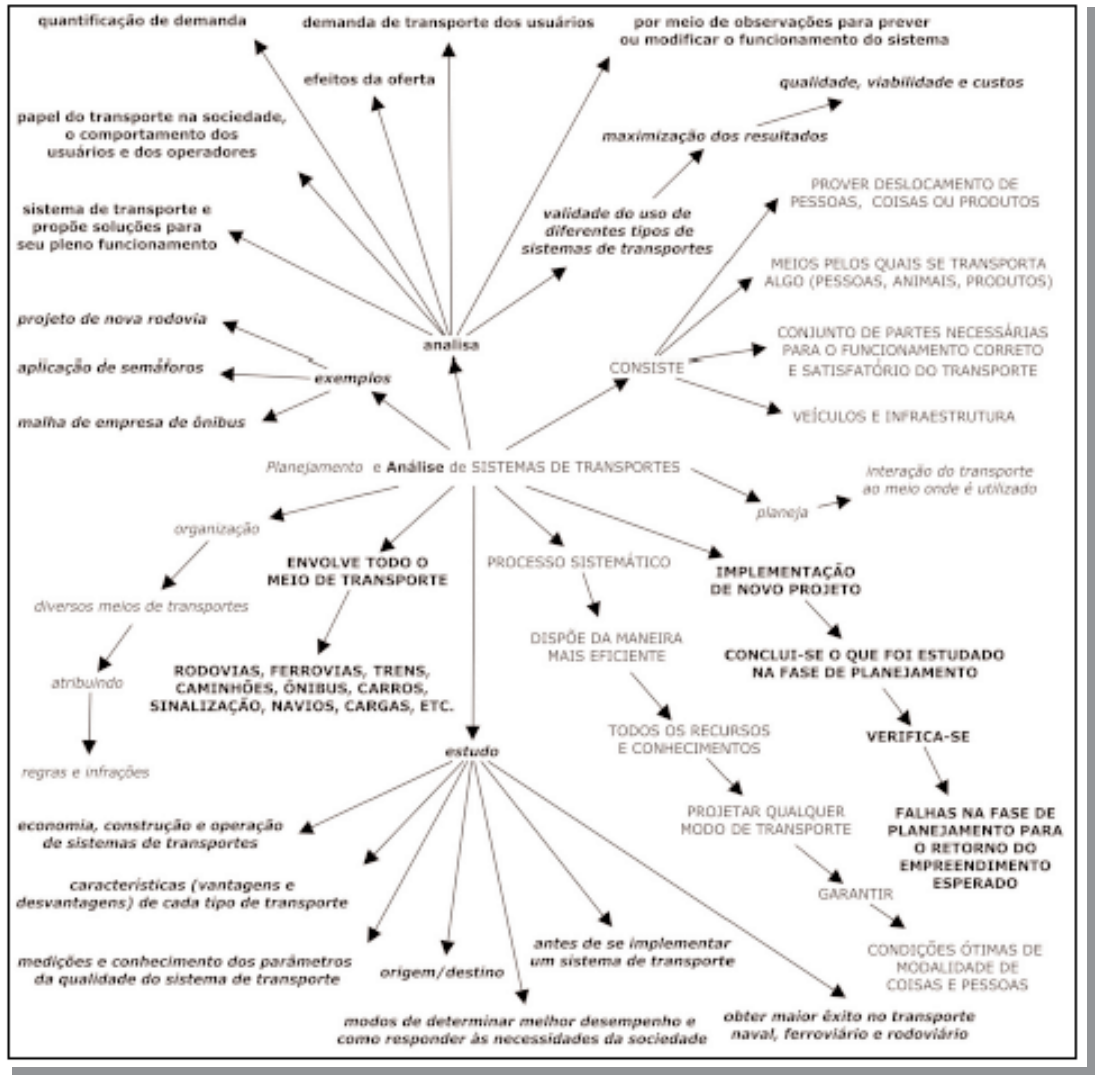

Figura 2 - Mapa cognitivo dos estudantes no método "tradicional"

Legenda: Planejamento (itálico)

Análise (negrito)

Sistemas (MAIÚSCULAS) 
As maneiras utilizadas pelos estudantes para solucionar as questões das partes 1 e 2 indicam o desenvolvimento de técnicas relacionadas ao PBL, apreendidas durante a experiência que envolveu a utilização do método na disciplina. Tais maneiras demonstram que os alunos tornaram-se progressivamente responsáveis por sua própria aprendizagem, tendo em vista que eles assumiram a responsabilidade na seleção das informações do domínio a ser explorado, decidindo o nível e a quantidade de informações a serem utilizadas. Ademais, esse resultado demonstra uma maior habilidade e maturidade em lidar com os problemas, indicando um maior comprometimento com o próprio aprendizado. Outro resultado observado está relacionado ao impacto que o PBL poderia causar aos alunos, em virtude do tempo de exposição em ambientes convencionais de ensino, afetando negativamente no aproveitamento até que desenvolvessem novos hábitos de estudos, como apontado por Albanese e Mitchell (1993) e Dochy et al. (2003). Contudo, esse impacto negativo não foi verificado na pesquisa; ao contrário, os estudantes envolvidos com as alternativas instrucionais demonstraram que, mesmo com o uso do método PBL obtiveram um bom aproveitamento, além de mostrarem-se mais interessados.

Por outro lado, quando se consideram as questões $2 a$ e $2 b$, referentes à aplicação prática dos conhecimentos apropriados na resolução dos problemas propostos, é possível notar diferenças significativas entre as respostas dos estudantes participantes e não participantes da nova proposta pedagógica, como apresentado nas Figuras 3 e 4. Observa-se, na Figura 3, uma maior riqueza de detalhes com o uso de procedimentos, sugestões, ideias, tempos e estimativas, sugerindo, de forma geral, um aproveitamento significativo dos estudantes participantes da experiência, tendo em vista 0 nível de abrangência e profundidade com que as questões foram respondidas. 


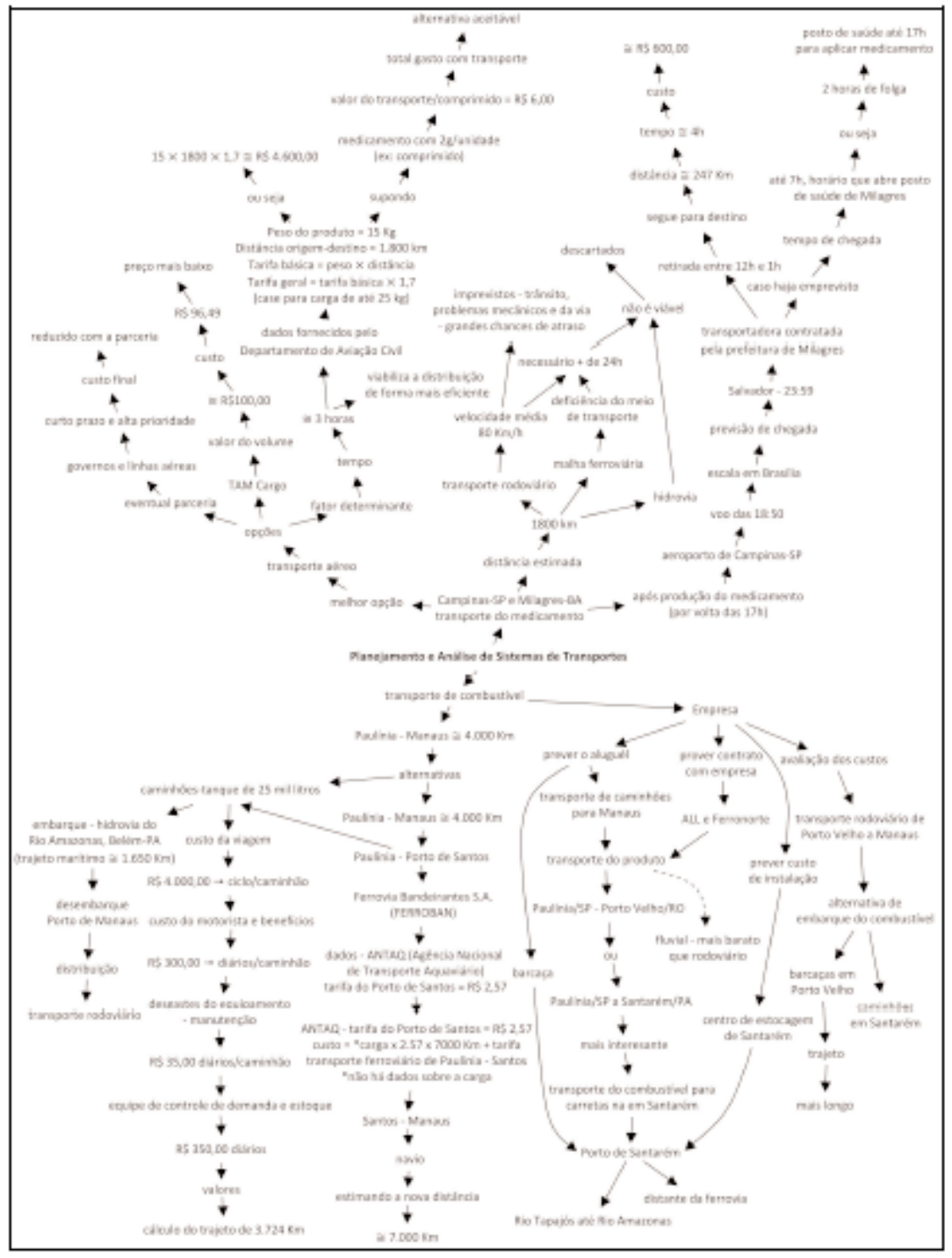

Figura 3 - Mapa cognitivo das respostas às questões 2a (parte superior da figura) e $2 b$ (parte inferior da figura) - Método PBL 
Observa-se ainda que, mesmo para as questões envolvendo a aplicação prática, os estudantes demonstraram apreensão efetiva dos conceitos trabalhados na disciplina - como se pode perceber pelas opções e possibilidades indicadas - tais como: meios de transportes viáveis, custos envolvendo a oferta e demanda dos produtos que fazem parte dos problemas abordados, melhores vias de acesso (sejam elas terrestres, aéreas ou marítimas), problemas para o transporte das mercadorias, deficiências, possíveis locais para embarque e desembarque, gastos com terceiros e desgaste de veículos. Os estudantes também realizaram pesquisas utilizando o acesso à Internet, buscando as informações que não sabiam, tais como: valores de transportes e de serviços fornecidos, distâncias percorridas entre uma localidade e outra, indicação de rotas com base em mapas rodoviários. Ademais, os alunos tiveram a iniciativa de entrar em contato com empresas especializadas em transporte de cargas, por meio de correio eletrônico, para obter informações que possibilitassem colocar em prática os conceitos e técnicas anteriormente aprendidas.

A exposição dos estudantes a respeito das questões abordadas, ou seja, as formas adotadas para solucionar os problemas, reflete $(\mathrm{m})$ de modo claro as características do método PBL. Esta constatação indica o desenvolvimento de certas habilidades relacionadas ao método, pois é possível perceber uma expressiva autonomia no processo de resolução: os alunos identificaram questões-chave, focaram seus esforços, indicaram os recursos. Deste modo, os alunos aplicaram seus conhecimentos, descobriram e explicaram para os colegas, desenvolveram novas habilidades sociais e cognitivas, responsabilidades e entendimentos, ou seja, aprenderam fazendo, conforme as considerações de Burch (2001) sobre a aprendizagem dos estudantes e as características do método PBL.

A comparação dos mapas construídos a partir das respostas dos estudantes submetidos às alternativas instrucionais com aquelas fornecidas pelos estudantes que aprenderam por meio do modelo convencional revela que, embora as duas turmas tenham procurado resolver os problemas propostos no questionário, as respostas dos estudantes submetidos ao PBL indicaram o uso dos conhecimentos e técnicas trabalhadas durante a experiência. 


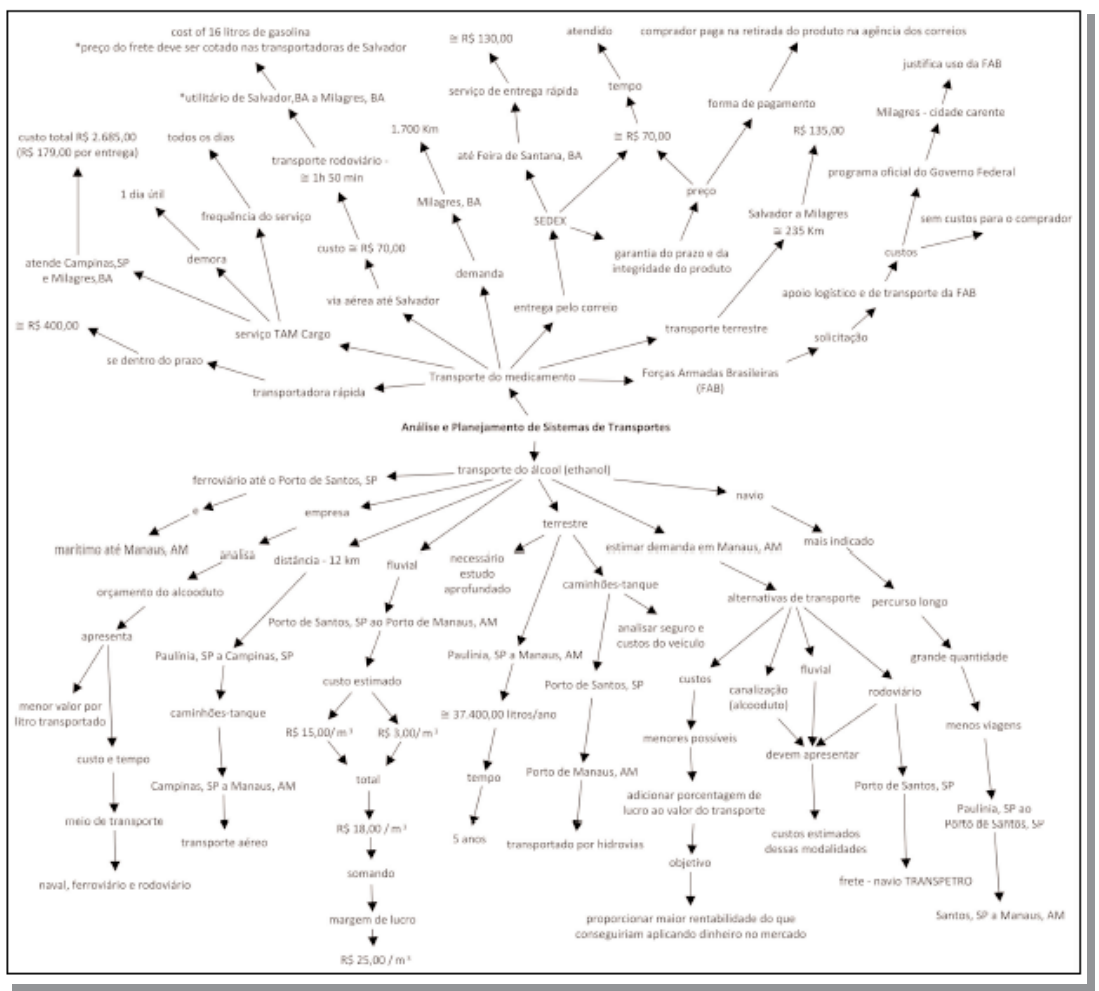

Figura 4 - Mapa cognitivo das respostas às questões 2a (parte superior da figura) e $2 b$ (parte inferior da figura) - Método "tradicional"

Podem notar-se certas diferenças não apenas na forma como as questões foram respondidas, isto é, nas técnicas utilizadas, mas nas soluções encontradas, que se mostraram viáveis para os problemas expostos, tanto de maneira geral quanto especificamente em relação às duas últimas questões, demonstradas nas Figuras 3 e 4.

Em síntese, embora os estudantes tenham sido expostos à experiência por curto período de tempo, o novo desenho instrucional proposto para a disciplina promoveu maiores níveis de aproveitamento e o desenvolvimento de comportamentos e atitudes desejáveis aos futuros profissionais, demonstrando a viabilidade do emprego do método PBL para ensinar Engenharia. Os resultados analisados e apresentados neste item, 
sobre o aproveitamento dos estudantes mediante sua experiência com o método PBL, ou seja, a nova proposta pedagógica, permitiram expressar algumas conclusões e considerações que serão abordadas na sequência.

\section{Conclusões e considerações finais}

Este estudo buscou investigar os efeitos do método PBL no aproveitamento dos estudantes de Engenharia Civil que participaram de uma experiência pedagógica na disciplina Planejamento e Análise dos Sistemas de Transporte, confrontando-o com o grupo de alunos que não participou de experiências inovadoras na disciplina.

A análise dos resultados obtidos com o questionário de avaliação do aprendizado, aplicado aos dois grupos de estudantes, mostrou efeitos significativos no aprendizado dos estudantes participantes da experiência com o uso do método PBL. Os efeitos positivos do método PBL relacionados ao desenvolvimento de técnicas de resolução de problemas apontam o comprometimento e responsabilidade dos alunos expostos à experiência em relação ao aprendizado, sugerindo o desenvolvimento da aprendizagem autônoma. Esta conclusão mostra uma diferença significativa entre o PBL e o método tradicional, uma vez que, embora o segundo possibilite realizar o cumprimento da tarefa de resolver os problemas propostos, de modo geral, ele se resumiu nesta tarefa sem, entretanto, haver o comprometimento com o aprendizado, desfavorecendo o desenvolvimento do pensamento crítico e criativo e a preocupação com o próprio aprendizado. Conforme Savery e Duffy (1995), como o ponto de convergência do PBL incide nos alunos, eles se tornam os construtores dos seus próprios conhecimentos. Nesse sentido, o fato de os alunos PBL manifestarem o comprometimento com a própria aprendizagem demonstra o desenvolvimento de comportamentos e atitudes diferenciadas, favoráveis ao exercício profissional, em um mercado que traz como marca principal a transformação.

O PBL mostrou ser um método viável para o ensino de Engenharia, sobretudo em cursos cujas disciplinas se baseiam, em sua maioria, em métodos convencionais de ensino. Deste modo, sua implementação não causou um impacto negativo no aproveitamento dos alunos, visto que eles se mostraram receptivos e envolvidos em métodos diferenciados de ensino- 
aprendizagem e inovações pedagógicas no ambiente instrucional, como aqui demonstrado e como anteriormente comprovado em diversos estudos (Kuri, Rodrigues da Silva \& Pereira, 2006; Kuri, Manzato \& Rodrigues da Silva, 2007; Kalatzis, 2008; Rodrigues da Silva, 2008). Ademais, o PBL proporcionou uma aprendizagem mais significativa e duradoura, outro aspecto positivo do método. Isso pôde ser constatado com o grupo de alunos que participou da experiência devido ao fato da atividade a que se refere o Quadro 1 ter ocorrido mais de um ano após o contato desses estudantes com as inovações instrucionais. Finalmente, este estudo sugere que a introdução de outros métodos ou alternativas pedagógicas no modo de ensinar e aprender em Engenharia pode proporcionar uma formação mais adequada ao perfil profissional que a sociedade hoje almeja, desde que conduzidos e avaliados com instrumentos e técnicas apropriadas.

\section{Referências}

Albanese, M. A., \& Mitchell, S. (1993). Problem-based learning: A review of literature on its outcomes and implementation issues. Academic Medicine, v. 68, n. 8, 52-81.

Barrows, H. S. (1996). Problem-based learning in medicine and beyond: A brief overview. In L. Wilkerson \& W. H. Gijselaers (Eds.), Bringing problem-based learning to higher education: Theory and practice (pp. 3-12). San Francisco: Jossey-Bass.

Barrows, H. S. (2008). Problem based learning initiative. IL: Southern Illinois University School of Medicine. Disponível em http://www.pbli.org/core.htm (acesso em 25 de Setembro de 2008).

Barrows, H. S., \& Myers, A. C. (1993). Problem-based learning in secondary schools. Springfield, IL: Problem-Based Learning Institute, Lanphier High School and Southern Illinois University Medical School.

Barrows, H. S., \& Tamblyn, R. (1976). An evaluation of problem-based learning in small groups using a simulated patient. Journal of Medical Education, v. 51, 52-54.

Barrows, H. S., \& Tamblyn, R. (1980). Problem-based learning: An approach to medical education. New York, USA: Springer Pub. Co.

Boud, D., \& Feletti, G. (Eds.). (1991). The challenge of problem-based learning. New York: St. Martin's Press.

Bridges, E. M., \& Hallinger, P. (1992). Problem-based learning for administrators. Oregon: ERIC Clearinghouse on Education Management, University of Oregon.

Burch, K. (2001). PBL, politics and democracy. In B. J. Duch, S. E. Groh \& D. E. Allen, The power of problem-based learning (pp. 193-205). Virginia: Stylus. 
Dochy, F., Segers, M., Van den Bossche, P., \& Gijbels, D. (2003). Effects of problembased learning: A meta-analysis. Learning and Instruction, n. 13, 533-568.

Duffy, T. M. (1994). Corporate and community education: Achieving success in the information society. Bloomington, IN: Indiana University.

Eden, C. L., \& Simpson, P. (1989). SODA and cognitive mapping in practice. In J. Rosenhead (Eds.), Rational analysis for a problematic world (pp. 43-70). London: John Wiley, Chichester.

Hassan, M. A. A, Yusof, K. M., Hamid, M. K. A., Hassim, M. H., Aziz, A. A., \& Hassan, S. A. H. S. (2004). A review and survey of problem-based learning application in engineering education. Conference on Engineering Education. Kuala Lumpur, Malaysia.

Kalatzis, A. C. (2008). Aprendizagem baseada em problemas em uma plataforma de ensino a distância com o apoio dos estilos de aprendizagem: Uma análise do aproveitamento dos estudantes de engenharia. Dissertação de Mestrado. São Carlos: Escola de Engenharia de São Carlos da Universidade de São Paulo.

Keating, S., \& Gabb, R. (2006). PBL in engineering student expectations in 2006. Postcompulsory Education Centre, Victoria University.

Kuri, N. P., Manzato, G. G., \& Rodrigues da Silva, A. N. (2007). Aprendizagem baseada em problemas em uma plataforma de ensino a distância: Uma aplicação do CoL na EESC-USP. Revista Minerva, v. 4, n. 1, 27-39.

Kuri, N. P., Rodrigues da Silva, A. N., \& Pereira, M. A. (2006). Estilos de aprendizagem e recursos da hipermídia aplicados no ensino de planejamento de transportes. Revista Portuguesa de Educação, v. 19, n. 2, 111-137.

Milter, R. G., \& Stinson, J. E. (1993). Educating leaders for the new competitive environment. In G. Gijselaers, S. Tempelaar \& Keiser, S. (Eds.), Educational innovation on economics and business administration: The case of problembased learning. London: Kluwer Academic Publishers.

Pawson, E., Fournier, E., Haigh, M., Muniz, O., Trafford, J., \& Vajoczki, S. (2006). Problem-based learning in Geography: Towards a critical assessment of its purposes, benefits and risks. Journal of Geography in Higher Education, v. 30, n. 1, 103-116.

Rehm, J. (1998). Problem-based learning: An introduction. The National Teaching and Learning Forum, v. 8, n. 1, 1-4.

Ribeiro, L. R. C. (2005). A aprendizagem baseada em problemas (PBL): Uma implementação na educação em engenharia na voz dos atores. Tese de Doutorado. São Carlos: Universidade Federal de São Carlos.

Rodrigues da Silva, A. N. (2008). Aprendizado baseado em problemas em uma plataforma de ensino a distância: Uma aplicação em engenharia. In III Ciclo de Palestras - O Ensino no Campus USP São Carlos: Inovações e inovadores. São Carlos: CETEPE/EESC-USP, CD-ROM.

Savery, J. R., \& Duffy, T. M. (1995). Problem-based learning: An instructional model and its constructivist framework. Educational Technology, n. 35, 31-41.

Subramaniam, R. M. (2006). Radiology: Problem-based learning - Concepts, theories, effectiveness and application to radiology teaching. Australian Radiology, v. 50, n. 4, 339-341. 
White, H. B. (1996). Dan tries problem-based learning: A case study. In L. Richlin (Eds.), To improve the academy (pp. 75-91). Stillwater, OK: New Forums Press and the Professional and Organizational Network in Higher Education.

Yusof, K. M., Tasir, Z., Harun, J., \& Helmi, S. A. (2005). Promoting problem-based learning (PBL) in engineering courses at the Universiti Teknologi Malaysia. Global Journal of Engineering Education, v. 9, n. 2, 175-184.

Yusof, K. M., Hassim, M. H., \& Azila, N. M. A. (2004). A first attempt at problem based learning in process dynamics and control course for chemical engineering undergraduates at Universiti Teknologi Malaysia. Proceedings 5th Asia Pacific Conference on Problem-Based Learning. Kuala Lumpur, Malaysia. 


\section{COGNITIVE MAPS IN PROBLEM BASED LEARNING EVALUATION}

\section{Abstract}

The purpose of this study is to investigate the learning performance of the Civil Engineering students using the Problem Based Learning (PBL) method as part of instructional innovations that took place in an Engineering Transportation course. We used the answers of the learning evaluation questionnaire to analyze the performance of the sample, which was submitted to the method, and the PBL effects resulted. This questionnaire contributed to evaluate and compare the learning of this sample with a group of students submitted to the traditional design. Cognitive maps were used as a strategy to investigate the effects of PBL and the occurrence of distinct learning performance among students. The results indicated that PBL students were engaged in their own learning, and their own performance. In addition, they achieved stronger behavior development and attitude, involvement, and a significant learning performance with the new design course.

Keywords

Problem Based Learning; Cognitive maps; Engineering education

\section{MAPAS COGNITIVOS EN LA EVALUACIÓN DEL APRENDIZAJE BASADO EN PROBLEMAS}

\section{Resumen}

El objetivo de este estudio es investigar el aprovechamiento de los estudiantes del curso de Ingeniería Civil en el que fue aplicado el método "Aprendizaje Basado en Problemas" (PBL) como parte de innovaciones pedagógicas implementadas en una asignatura del area de Ingeniería de Transportes. Para analisar el aprovechamiento de la muestra sometida a las alternativas instruccionales fueron utilizadas las respuestas de los estudiantes 
a un cuestionario para evaluación del aprendizaje elaborado por el profesor. Este instrumento contribuyó para evaluar y comparar el aprendizaje de la muestra con un grupo de alumnos sometidos al modelo tradicional de enseñanza. Las respuestas fueron agrupadas y analisadas por medio de mapas cognitivos, contribuyendo con la investigación de los efectos del método PBL y de la ocurrencia de aprovechamientos diferenciados entre los alumnos. Los resultados indican que los estudiantes obtuvieron mejor envolvimiento, desarrollos de comportamientos y actitudes, compromiso con el propio aprendizaje y un aprovechamiento significativamente mejor con el nuevo diseño de la asignatura.

Palabras clave

Aprendizaje basado en problemas; Mapas cognitivos; Enseñanza en Ingeniería

Recebido em Junho, 2009

Aceite para publicação em Maio, 2011

\footnotetext{
Toda a correspondência relativa a este artigo deve ser enviada para: Adriana Casale, Escola de Engenharia de São Carlos, Departamento de Engenharia de Produção, Universidade de São Paulo, Avenida Trabalhador São-Carlense, 400, CEP 13560-590, São Carlos, São Paulo, Brasil. E-mail: dricasale@usp.br
} 